\title{
Insights from individuals successfully recovered from cannabis use disorder: natural versus treatment-assisted recoveries and abstinent versus moderation outcomes
}

\author{
David C. Hodgins ${ }^{*}(\mathbb{D}$ and Jonathan N. Stea
}

\begin{abstract}
Background: Increasing understanding of the pathways and processes of recovery from cannabis use disorder may help in designing effective and attractive interventions to promote recovery. We report insights from individuals who had successfully recovered from cannabis use disorder with a variety of pathways. Recovered individuals describe their perceptions of why they developed the problem, why they were successful in recovering, and the advice they would offer to individuals with similar problems.
\end{abstract}

Methods: Media announcements were used to recruit 119 volunteers who met lifetime but not past year criteria for cannabis use disorder. Participants were asked open-ended questions which were content analyzed and compared between individuals who whose recoveries were treatment-assisted (45\%) versus natural (55\%) and between individuals who were abstinent (57\%) versus those who continued non-problematic consumption (43\%).

Results: Participants most frequently described their problems as having developed due to the use of cannabis to cope, because of environmental and social influences, and enjoyment of the positive effects. Success in recovery was attributed to focusing on reasons for change, goal commitment to change, and conquering denial/self-deception. Treatment-assisted participants were more likely to perceive that they overcame their cannabis problem due to treatment/ self-help and conquering underlying issues, whereas naturally recovered participants were more likely to describe focusing on reasons for change, will power, and lost enjoyment/lifestyle change. Treatment-assisted participants were more likely to recommend seeking help/social support and naturally recovered participants were more likely to endorse reflecting on reasons for change, engaging in hobbies/distracting activities, and stimulus control/avoidance/change social environment. The majority recommended professional treatment (79.1\%) and self-help materials (76.9\%), and a little over half (53.2\%) would also recommend natural recovery.

Conclusions: These insights from people with lived experience further support previous research that treatmentassisted and natural recoveries are for the most part similar with respect to the recovery process. However, participants, whether or not they had had treatment involvement, recommended the use of treatment and self-help materials to sharpen their focus on the reasons to change and to enhance their commitment to change. At the same time, they saw value in the efforts of individuals to recovery without help.

Keywords: Cannabis, Marijuana, Cannabis use disorder, Natural recovery, Treatment, Recovery pathways

*Correspondence: dhodgins@ucalgary.ca

Department of Psychology, University of Calgary, 2500 University Drive

NW, Calgary, AB T2N 1N4, Canada 


\section{Background}

Rates of cannabis use and cannabis use disorder (CUD) are rising in North America, and CUD is a common presenting issue in addiction treatment [20,32]. However, treatment-seeking rates are low relative to the numbers of people with the disorder [17] and rates of natural recovery are significant $[3,8]$. Increasing our understanding of the pathways and processes of recovery may help in designing effective and attractive interventions to promote recovery.

A growing body of literature supports the efficacy of a variety of interventions for CUD [14]. However, only a few empirical studies of the recovery process from CUDs have been reported. Cunningham et al. [9] interviewed former frequent cannabis users about their reasons for stopping. The most common explanations included emotional maturation and taking on new roles and responsibilities. Boyd et al. [4] interviewed cannabis users who had quit using at least once in the past about change strategies. Participants described changing their environment to be the most helpful strategy and help from professionals as the least helpful strategy. Ellingstad interviewed a more defined group of former daily cannabis users who had quit for at least 1 year without the assistance of treatment [11]. The major reasons described for resolving their problem were increasingly viewing cannabis as negative, experiencing more negative effects, and change being related to a broader lifestyle shift. Strategies cited as helpful in quitting included involvement in activities unrelated to cannabis use, avoidance of triggers to use, and lifestyle changes. Finally, in the only prospective study, Swift et al. [30] followed a group of 200 longterm cannabis users for a 1-year period during which time nearly two-thirds attempted to reduce or stop using. The top reasons for these change attempts were physical or psychological health effects, boredom with using, concerns they were using too much, lack of money, and lifestyle circumstances.

One limitation of the existing research is that samples generally include individuals with relatively brief periods of reduction or cessation of use, and therefore, the results may not be relevant for understanding the longer-term recovery process, which typically occurs after multiple change attempts. The only study that focused on stable recoveries, specifically, is the Ellingstad study [11] of abstinent individuals who had naturally recovered (i.e., did not attend treatment). We extended this research by comparing the experiences of individuals who had recovered with and without the assistance of treatment, either though abstinence from any use or moderation of use [29]. The treatment-assisted and abstinence groups exhibited higher levels of lifetime severity of cannabis problems than the natural and moderation groups. This association between severity of problem and recovery pathways has been found for other addictive behaviours including alcohol, heroin, and gambling [3]. Interestingly, however, despite the different pathways to recovery, the motivators and processes of recovery were mostly similar for the different groups. Regardless of whether they sought treatment or not, or whether they become abstinent or moderated their use, recovered individuals most frequently described their motivations for tackling recovery as a conscious choice that was related to their cannabis use becoming incompatible with their self-image and lifestyle, and related to their use causing negative psychological concerns. They described relying most frequently on cognitive strategies such as thinking about the negative consequences of their use or the benefits of less use as well as a variety of behavioural strategies (decreasing time spent with other users, avoidance of cues to use, involvement in incompatible activities).

These descriptions of pathways and processes of recovery are potentially helpful in the development of treatment approaches and treatment system policies. For example, the association between severity and treatment pathway is consistent with the tenets of stepped care models that offer increasingly intense treatment for increasingly severe problems. However, given that the treatment processes appear similar in the different pathways, it is important to delve deeper into individual experiences for a more complete understanding of the recovery process. In the present report we describe further insights from individuals who have successfully recovered from cannabis use disorder, contrasting treatment-assisted and natural pathways, and abstinent and moderation outcomes. Comparing these groups potentially provides the broad perspective necessary to conceptualize treatment systems. Specifically, we summarize individual perceptions of why they developed the problem, why they were successful in recovering, and the advice they would offer to individuals with similar problems. The perspective of people with lived experience is increasingly recognized as central to planning interventions that are both effective and attractive [24].

\section{Methods}

\section{Participants}

Media announcements were used to recruit 119 volunteers for an in-person interview who met lifetime but not past year criteria for cannabis use disorder [2], according to the Composite International Diagnostic Interview (CIDI) [21]. Recruitment ads asked for individuals who were willing to share information about their recovery, which might be helpful in designing future treatment programs for other people. Other inclusion criteria were age 18 years and older, ability to read and write English, 
and willingness to refrain from any alcohol or illicit drug use for at least $8 \mathrm{~h}$ prior to the interview. In addition, participants were asked to provide, if possible, the name of a family member or friend who would be able to corroborate their cannabis use history.

The sample was $30 \%$ female and $70 \%$ male with a mean age of 37.3 years. The mean age of first use of cannabis was 14.7 years $(\mathrm{SD}=3.0)$ and the mean age of onset of CUD was 20.0 years $(\mathrm{SD}=6.5)$. The median length of recovery was 5 years. A history of treatment was reported by 53 individuals (45\%). Sixty-eight (57\%) were categorized as abstinent (no cannabis use for at least 12 months) and 51 (43\%) reported some non-problematic use. Individuals reporting use most frequently reported using once or twice in the past 12 months (59\%) or monthly $(20 \%)$, although $6 \%$ reported weekly use and $16 \%$ almost daily use. Other demographic details are provided in Stea et al. [29] and Additional file 1: Table S1.

\section{Interview}

As the final section of a longer in-person interview that queried in detail cannabis use history, mental health comorbidity, and recovery efforts, and strategies, participants were asked a series of open-ended questions about the recovery process. Responses were probed for clarity and were recorded in writing by the interviewer. A number of areas were queried. First participants were asked: "What advice would you give to help another person with a marijuana problem"? Next they were asked whether they would recommend another person to reduce use to non-problematic levels or to quit completely (and why), and whether they would recommend that another person seek professional help or self-help materials or overcome the problem on their own without professional assistance. Participants were asked to describe their understanding of why they personally developed a marijuana problem, and finally, they were asked to describe their understanding of why they were able to overcome their problem. Participants could provide multiple responses to each question.

\section{Data analysis}

Responses were content analysed [5] with the aid of NVivo software [25]. An inductive approach was used as no a priori hypotheses were proposed. One individual derived categories reflecting responses to each of the above questions (JS), and then an independent rater used the category labels and a brief one sentence category description to code each response. Inter-rater reliability was assessed using percentage agreement and Kappa coefficients. Kappa greater than .80 was set as the minimum level of agreement a priori. Disagreements were resolved through discussion to provide a final categorization of each response. Frequency counts were calculated for the total sample, and for abstinence versus moderation subgroups (recovery use status), and the treatment-assisted versus natural recovery subgroups (recovery pathway), which were compared using Chi squared tests, with Bonferroni correction to the alpha level according to the number of categories. In the case where a significant result was obtained on a particular dependent variable for both recovery use status and recovery pathway, follow-up tests were conducted that controlled for the effects of each respective factor in order to elucidate the relationship with the dependent variable.

\section{Results}

\section{Perceived etiology}

Ten categories of perceived personal etiology were uncovered and interrater reliability was excellent (percent agreement $=89.8$, Kappa $=.88)$. Participants identified a mean of 1.6 $(\mathrm{SD}=0.7)$ categories and group comparisons revealed no differences between the abstinence- $(M=1.7$, $S D=0.7)$ and moderation $(M=1.6, S D=0.8)$ recovery use status groups, $F(1,115)=0.1, n s$, or between the treatment-assisted $(M=1.7, S D=0.8)$ and natural recovery $(M=1.6, S D=0.7)$ pathways groups, $F(1,115)=0.4$, $n s$. Table 1 displays the percentage of participants that endorsed each category (Additional file 1: Table S2 provides category descriptions). The top three categories reported were: used cannabis to cope (43.7\%), environment/social influence (41.2\%), and enjoyment/boredom/ positive perceptions of cannabis (23.5\%). The category of used cannabis to cope reflected participant responses wherein cannabis was used to escape, self-medicate, and avoid emotional problems. The category of environment/social influence reflected participant responses wherein peer pressure and being around cannabis users was thought to contribute to the development of the cannabis problem. The category of enjoyment/boredom/ positive perceptions of cannabis included enjoyment of the high, boredom, and positive perceptions of cannabis being helpful or fun in some way (e.g., mind enhancing/ expanding, creating a philosophical and/or silly environment) was thought to contribute to the development of the cannabis problem.

While no differences emerged between the recovery use status, three differences emerged between the recovery pathway. Specifically, the treatment-assisted group was more likely to endorse the categories of used cannabis to cope (54.7 vs. $34.8 \%)$ and genetics/predisposition (20.8 vs. $1.5 \%$ ), whereas the natural recovery group was more likely to endorse the category of enjoyment/boredom/ positive perceptions of cannabis (31.8 vs. $13.2 \%)$. With a Bonferroni correction for 10 comparisons $(\alpha=.005)$, only 
Table 1 Percentage of participants that endorsed perceived etiology categories for the total sample and group comparisons

\begin{tabular}{|c|c|c|c|c|c|c|c|}
\hline \multirow[t]{2}{*}{ Category (\%) } & \multirow{2}{*}{$\begin{array}{l}\text { Total sample } \\
(N=119)\end{array}$} & \multicolumn{3}{|c|}{ Recovery use status } & \multicolumn{3}{|c|}{ Recovery pathway } \\
\hline & & $\mathrm{AB}(n=68)$ & $\operatorname{MOD}(n=51)$ & $x^{2}$ & $\operatorname{TAR}(n=53)$ & $\mathrm{NR}(n=66)$ & $x^{2}$ \\
\hline Used cannabis to cope & 43.7 & 47.1 & 39.2 & 0.7 & 54.7 & 34.8 & $4.7^{*}$ \\
\hline Environment/social influence & 41.2 & 38.2 & 45.1 & 0.6 & 39.6 & 42.4 & 0.1 \\
\hline $\begin{array}{l}\text { Enjoyment/boredom/positive perceptions } \\
\text { of cannabis }\end{array}$ & 23.5 & 20.6 & 27.5 & 0.8 & 13.2 & 31.8 & $5.7^{*}$ \\
\hline Addictive personality & 14.3 & 11.8 & 17.6 & 0.8 & 17.0 & 12.1 & 0.6 \\
\hline Genetics/predisposition & 10.1 & 14.7 & 3.9 & 3.7 & 20.8 & 1.5 & $12.0^{* * *}$ \\
\hline Habit/dependence/addiction & 9.2 & 11.8 & 5.9 & $n s^{i}$ & 7.5 & 10.6 & $n s^{i}$ \\
\hline Loss of control & 7.6 & 8.8 & 5.9 & $n s^{i}$ & 9.4 & 6.1 & $n s^{i}$ \\
\hline Cannabis per se causes the addiction & 6.7 & 5.9 & 7.8 & $n s^{i}$ & 1.9 & 10.6 & $n s^{i}$ \\
\hline No problem actually existed & 4.2 & 5.9 & 2.0 & $n s^{i}$ & 0.0 & 7.6 & $n s^{i}$ \\
\hline Denial/self-deception/ignorance/choice & 3.4 & 1.5 & 5.9 & $n s^{i}$ & 3.8 & 3.0 & $n s^{i}$ \\
\hline
\end{tabular}

Chi square values represent Pearson Chi square values. Absolute Chi square values are reported. $A B$ abstinence; $M O D$ moderation; NR natural recovery; TAR treatmentassisted recovery

' Fisher's exact test was used instead of Pearson Chi square because expected cell counts were less than 5

${ }^{*} p<.05 ;{ }^{* *} p<.01 ;{ }^{* * *} p<.001$

the difference between the treatment-assisted group and the natural recovery group on the genetics/predisposition category remained statistically significant.

\section{Perceived causes of recovery success}

Twelve categories of participants' perceived causes of their recovery success showed excellent inter-rater reliability (percentage agreement $=83.3 \%$, Kappa $=.81$, see Additional file 1: Table S3 for category descriptions). Participants identified a mean of $1.5(S D=0.8)$ categories. Group comparisons on the mean number of categories revealed no differences between the abstinence- $(M=1.5$, $S D=0.7)$ and moderation $(M=1.6, S D=0.8)$ recovery use status groups, $F(1,115)=0.1, n s$, or between the treatment-assisted $(M=1.5, S D=0.7)$ and natural recovery $(M=1.6, S D=0.8)$ pathways, $F(1,115)=0.2$, ns. As can be seen in Table 2, the top three major categories reported by the total sample were: focused on reasons for change (36.1\%), goal commitment to change (31.9\%), and conquered denial/self-deception (25.2\%). The category of focused on reasons for change reflected participant responses wherein recovery success was attributed to thinking about reasons for change and goal pursuit. The category of goal commitment to change reflected participant responses wherein recovery success was attributed to having strong motivation and commitment to change. The category of conquered denial/self-deception reflected participant responses wherein recovery success was attributed to a found sense of self-awareness or realization that cannabis was a problem.
While no differences emerged between the recovery use status groups, five differences emerged between the recovery pathways groups. Specifically, the treatmentassisted group was more likely to endorse the categories of treatment/self-help (24.5 vs. $4.5 \%)$ and conquered underlying issues (11.3 vs. 0.0\%), whereas the natural recovery group was more likely to endorse the categories of focused on reasons for change (47.0 vs. $22.6 \%)$, will power (15.2 vs. $3.8 \%$ ), and lost enjoyment/lifestyle change (13.6 vs. $1.9 \%$ ). With a Bonferroni correction for $12 \mathrm{com}$ parisons $(\alpha=.004)$, only the difference between the treatment-assisted group and the natural recovery group on the treatment/self-help category remained statistically significant.

\section{Recovery advice \\ Strategies}

Content analysis was used to derive thirteen categories of hypothetical advice provided by participants to help another person with a cannabis problem. Excellent inter-rater reliability was obtained (percentage agreement $=91.0 \%$, Kappa $=.90$, see Additional file 1: Table S4 for category descriptions). Participants identified a mean of $1.8(S D=1.7)$ pieces of advice, with no differences between the abstinence- $(M=1.9$, $S D=0.9)$ and moderation $(M=1.7, S D=0.9)$ use status groups, $F(1,115)=2.3$, ns, or between the treatment-assisted $(M=1.7, S D=0.9)$ and natural recovery $(M=1.9, S D=0.9)$ groups, $F(1,115)=0.8$, ns. As can be seen in Table 3, the top three major advice categories reported by the total sample were: seek help/social 
Table 2 Percentage of participants that endorsed perceived causes of recovery success categories for the total sample and group comparisons

\begin{tabular}{|c|c|c|c|c|c|c|c|}
\hline \multirow[t]{2}{*}{ Category (\%) } & \multirow{2}{*}{$\begin{array}{l}\text { Total sample } \\
(N=119)\end{array}$} & \multicolumn{3}{|c|}{ Recovery use status } & \multicolumn{3}{|c|}{ Recovery pathway } \\
\hline & & $\mathrm{AB}(n=68)$ & $\operatorname{MOD}(n=51)$ & $x^{2}$ & $\operatorname{TAR}(n=53)$ & NR $(n=66)$ & $x^{2}$ \\
\hline Focused on reasons for change & 36.1 & 35.3 & 37.3 & 0.0 & 22.6 & 47.0 & $7.5^{* *}$ \\
\hline Goal commitment to change & 31.9 & 30.9 & 33.3 & 0.1 & 35.8 & 28.8 & 0.7 \\
\hline Conquered denial/self-deception & 18.5 & 14.7 & 23.5 & 1.5 & 18.9 & 18.2 & 0.0 \\
\hline Treatment/self-help & 13.4 & 16.2 & 9.8 & 1.0 & 24.5 & 4.5 & $10.1^{* * *}$ \\
\hline Religious/spiritual guidance & 12.6 & 14.7 & 9.8 & 0.6 & 13.2 & 12.1 & 0.0 \\
\hline Will power & 10.1 & 13.2 & 5.9 & 1.7 & 3.8 & 15.2 & $4.2^{*}$ \\
\hline Lost enjoyment/lifestyle change & 8.4 & 5.9 & 11.8 & $n s^{i}$ & 1.9 & 13.6 & $s^{* i}$ \\
\hline Social support & 8.4 & 7.4 & 9.8 & $n s^{i}$ & 11.3 & 6.1 & $n s^{i}$ \\
\hline $\begin{array}{l}\text { Stimulus control/avoidance/changed } \\
\text { social environment }\end{array}$ & 5.9 & 4.4 & 7.8 & $n s^{i}$ & 5.7 & 6.1 & $n s^{i}$ \\
\hline Conquered underlying issues & 5.0 & 7.4 & 2.0 & $n s^{i}$ & 11.3 & 0.0 & $s^{* * i}$ \\
\hline Luck/lack of cravings or withdrawal & 3.4 & 1.5 & 5.9 & $n s^{i}$ & 3.8 & 3.0 & $n s^{i}$ \\
\hline Helping others & 1.7 & 2.9 & 0.0 & $n s^{i}$ & 0.0 & 3.0 & $n s^{i}$ \\
\hline
\end{tabular}

Chi square values represent Pearson Chi square values. Absolute Chi square values are reported. $A B$ abstinence; $M O D$ moderation; NR natural recovery; TAR treatmentassisted recovery

' Fisher's exact test was used instead of Pearson Chi square because expected cell counts were less than 5

${ }^{*} p<.05 ;{ }^{* *} p<.01 ;{ }^{* * *} p<.001$

Table 3 Percentage of participants that endorsed advice for the total sample and group comparisons

\begin{tabular}{|c|c|c|c|c|c|c|c|}
\hline \multirow[t]{2}{*}{ Category (\%) } & \multirow{2}{*}{$\begin{array}{l}\text { Total sample } \\
(N=119)\end{array}$} & \multicolumn{3}{|c|}{ Recovery use status } & \multicolumn{3}{|c|}{ Recovery pathway } \\
\hline & & $\mathrm{AB}(n=68)$ & $\operatorname{MOD}(n=51)$ & $x^{2}$ & $\operatorname{TAR}(n=53)$ & $\mathrm{NR}(n=66)$ & $x^{2}$ \\
\hline Seek help/social support & 37.8 & 47.1 & 25.5 & $5.8^{*}$ & 64.2 & 16.7 & $28.2^{* * *}$ \\
\hline Reflect on reasons for change & 26.1 & 22.1 & 31.4 & 1.3 & 17.0 & 33.3 & $4.1^{*}$ \\
\hline Engage in hobbies/distracting activities & 25.2 & 23.5 & 27.5 & 0.2 & 15.1 & 33.3 & $5.2^{*}$ \\
\hline $\begin{array}{l}\text { Stimulus control/avoidance/change social } \\
\text { environment }\end{array}$ & 21.8 & 19.1 & 25.5 & 0.7 & 13.2 & 28.8 & $4.2^{*}$ \\
\hline Think positively & 14.3 & 14.7 & 13.7 & 0.0 & 9.4 & 18.2 & 1.8 \\
\hline Face denial/self-deception & 13.4 & 16.2 & 9.8 & 1.0 & 13.2 & 13.6 & 0.0 \\
\hline Change is a personal decision & 11.8 & 13.2 & 9.8 & 0.3 & 7.5 & 15.2 & $n s^{\mathrm{i}}$ \\
\hline Find underlying issue/motive for use & 9.2 & 8.8 & 9.8 & $n s^{i}$ & 13.2 & 6.1 & $n s^{i}$ \\
\hline Quit & 7.6 & 10.3 & 3.9 & $n s^{i}$ & 5.7 & 9.1 & $n s^{i}$ \\
\hline Research cannabis/addiction & 5.0 & 4.4 & 5.9 & $n s^{\mathrm{i}}$ & 5.7 & 4.5 & $n s^{\mathrm{i}}$ \\
\hline Seek spiritual/religious guidance & 5.0 & 7.4 & 2.0 & $n s^{\mathrm{i}}$ & 3.8 & 6.1 & $n s^{\mathrm{i}}$ \\
\hline Moderate use & 2.5 & 4.4 & 0.0 & $n s^{\mathrm{i}}$ & 3.8 & 1.5 & $n s^{\mathrm{i}}$ \\
\hline Not sure/no answer & 2.5 & 1.5 & 3.9 & $n s^{i}$ & 5.7 & 0.0 & $n s^{i}$ \\
\hline
\end{tabular}

Chi square values represent Pearson Chi square values. Absolute Chi square values are reported. $A B$ abstinence; $M O D$ moderation; NR natural recovery; TAR treatmentassisted recovery

' Fisher's exact test was used instead of Pearson Chi square because expected cell counts were less than 5

${ }^{*} p<.05 ;{ }^{* *} p<.01 ;{ }^{* * *} p<.001$

support (37.8\%), reflect on reasons for change (26.1\%), and engage in hobbies/distracting activities (25.2\%). The category of seek help/social support reflected advice to seek a variety of forms of help, including from friends, family, and/or formal or professional help. The category of reflect on reasons for change included advice to think about the negative consequences of cannabis use, the reasons to change, and how life can be better without cannabis. The category of engage in hobbies/distracting 
activities reflected advice to occupy oneself via the pursuit of goals, hobbies, and other activities.

Several differences emerged among the groups. With respect to recovery status type, there was only one difference; the abstinence-oriented group was more likely to endorse the category of seek help/social support compared to the moderation-oriented group (47.1 vs. $25.1 \%$ ); however, when recovery pathway was controlled for, there was no longer a significant relationship [treatmentassisted participants, $\chi^{2}(1)=3.2, n s$; naturally recovered participants, $\left.\chi^{2}(1)=0.2, n s\right]$. With respect to recovery pathway, the treatment-assisted group was more likely to endorse the category of seek help/social support (64.2 vs. $16.7 \%$ ) - this relationship remained significant after controlling for recovery use status [treatment-assisted participants, 72.2 vs. $18.8 \%, \mathrm{X}^{2}(1)=19.4, p<.001$; naturally recovered participants, 47.1 vs. $14.7 \%$, Fisher's exact test, sig.] - whereas the natural recovery group was more likely to endorse the categories of reflect on reasons for change (33.3 vs. 17.0\%), engage in hobbies/distracting activities (33.3 vs. 15.1\%), and stimulus control/avoidance/ change social environment (28.8 vs. $13.2 \%)$. With a Bonferroni correction for 13 comparisons $(\alpha=.004)$, only the difference between the treatment-assisted group and the natural recovery group on the seek help/social support category remained statistically significant.

\section{Pathways}

Participants were also asked to provide a number of recommendations about different recovery pathways, the results of which are displayed in Table 4. The majority of the total sample reported that they would recommend professional treatment (79.1\%) and self-help materials $(76.9 \%)$, and a little over half $(53.2 \%)$ would recommend natural recovery (i.e., that a hypothetical person with a cannabis problem try to overcome the problem on their own without professional assistance). Interestingly, the moderation status group was significantly more likely to recommend natural recovery than the abstinent group (67.4 vs. $42.9 \%$ ); however, when recovery pathway was controlled for, this relationship only remained significant among treatment-assisted participants (53.3 vs. $20.0 \%$, Fisher's exact test, sig.), not naturally recovered participants $\left[\mathrm{X}^{2}(1)=0.0, n s\right]$. With respect to the recovery pathway group, the treatment-assisted group was significantly more likely to recommend self-materials

Table 4 Participant recommendations for the total sample and group comparisons

\begin{tabular}{|c|c|c|c|c|c|c|c|}
\hline \multirow[t]{2}{*}{ Variable } & \multirow{2}{*}{$\begin{array}{l}\text { Total } \\
\text { sample } \\
(N=119)\end{array}$} & \multicolumn{3}{|c|}{ Recovery use status } & \multicolumn{3}{|c|}{ Recovery pathway } \\
\hline & & $\mathrm{AB}(n=68)$ & $\operatorname{MOD}(n=51)$ & $x^{2}$ & $\operatorname{TAR}(n=53)$ & NR $(n=66)$ & $x^{2}$ \\
\hline $\begin{array}{l}\text { Recommend professional treatment for cannabis problem ( } \% \\
\text { yes) }\end{array}$ & $79.1^{\mathrm{a}}$ & $79.1^{\mathrm{b}}$ & $79.2^{c}$ & 0.0 & $86.5^{\mathrm{d}}$ & $73.0^{\mathrm{e}}$ & 3.1 \\
\hline Recommend self-help materials for cannabis problem (\% yes) & $76.9^{f}$ & 79.4 & $73.5^{9}$ & 0.6 & 86.8 & $68.8^{h}$ & $5.3^{*}$ \\
\hline Recommend natural recovery for cannabis problem (\% yes) & $53.2^{j}$ & $42.9^{e}$ & $67.4^{k}$ & $6.4^{*}$ & $30.0^{1}$ & $72.9^{\mathrm{m}}$ & $20.0^{* * *}$ \\
\hline \multicolumn{8}{|l|}{ Recommend $A B$ versus $M O D(\%)$} \\
\hline Quit & 48.7 & 58.8 & 35.3 & $6.5^{*}$ & 62.3 & 37.9 & $7.0^{* *}$ \\
\hline Depends on the person & 24.4 & 19.1 & 31.4 & 2.4 & 17.0 & 30.3 & 2.8 \\
\hline Reduce/cut-back & 19.3 & 13.2 & 27.5 & $3.8^{*}$ & 15.1 & 22.7 & 1.1 \\
\hline Neither or both & 7.6 & 8.8 & 5.9 & $n s^{i}$ & 5.7 & 9.1 & $n s^{i}$ \\
\hline
\end{tabular}

Chi square values represent Pearson Chi square values. Absolute Chi square values are reported. $A B$ abstinence; $M O D$ moderation; $N R$ natural recovery; TAR treatmentassisted recovery

' Fisher's exact test was used instead of Pearson Chi square because expected cell counts were less than 5

a $n=115$

b $n=67$

c $n=48$

d $n=52$

e $n=63$

f $n=117$

g $n=49$

h $n=64$

j $n=109$

${ }^{k} n=46$

I $n=50$

${ }^{m} n=59$

${ }^{*} p<.05 ;{ }^{* *} p<.01 ;{ }^{* * *} p<.001$ 
(86.8 vs. $68.8 \%$ ), whereas the natural recovery group was significantly more likely to recommend natural recovery (72.9 vs. $30.0 \%$ ); however, when recovery use status was controlled for, this latter relationship only remained significant among abstinence-oriented participants [71.4 vs. $\left.20.0 \%, X^{2}(1)=16.8, p<.001\right]$, not moderation-oriented participants (Fisher's exact test, $n s$ ).

When asked whether a person should reduce/cut-back or quit their cannabis use completely, the most popular response was to quit (48.7\%), followed by depends on the person (24.4\%), reduce/cut back (19.3\%), and neither or both $(7.6 \%)$. The abstinence-oriented group was significantly more likely to recommend that people quit (58.8 vs. $35.3 \%)$; however, when recovery pathway was controlled for, this relationship no longer remained significant [treatment-assisted participants, $\chi^{2}(1)=2.5$, $n s$; naturally recovered participants, $\left.\chi^{2}(1)=2.1, n s\right]$. Moreover, the moderation status group was significantly more likely to recommend that people reduce/cut-back (27.5 vs. $13.2 \%$ ). With respect to recovery pathway, the treatment-assisted group was significantly more likely to recommend that people quit compared to the natural recovery group (62.3 vs. $37.9 \%$ ); however, when recovery status was controlled for, this relationship no longer remained significant [abstinence-oriented participants, $X^{2}(1)=3.6, n s ;$ moderation-oriented participants, $X^{2}$ $(1)=1.5, n s]$.

\section{Discussion}

Understanding the perceptions of people with lived experience about the development and resolution of a CUD is instructive in conceptualizing and promoting the process of recovery. Individuals with stable recoveries are one important informant group, but it is important that different experiences be represented, including different pathways and both abstinent and moderation outcomes. In this study, recovered individuals described their problems as having developed for multiple reasons, but in particular due to the use of cannabis to cope, because of environmental and social influences, and enjoyment of the positive effects. These perceptions align with research into the etiology of CUD insofar as coping motives for cannabis use have been found to be associated with the development of cannabis use disorders [6, 13]; environmental and social influences such as low parental monitoring, high peer group deviance, and cannabis availability have been found to predict cannabis initiation [15]; and perceived risk of harm has been found to be inversely associated with frequency of cannabis use $[10,12,19]$. Interestingly, we previously reported the responses of this sample to the Marijuana Motives Measure (MMM, [27]), which indicated that participants most often used cannabis for enhancement motives during their lifetime, followed by social, coping, expansion, and conformity motives [29]. This suggests that while participants believed that coping motives were the strongest contributor to the development of their cannabis problem, they more often used cannabis for other purposes (i.e., enhancement and social reasons), which highlights the pervasiveness of cannabis use during their lifetime.

Treatment-assisted participants were more likely to endorse genetics/predisposition compared to naturally recovered participants, whereas the latter group was more likely to endorse the reason of enjoyment/boredom/ positive perceptions of cannabis. The fact that treatmentassisted participants more readily identified genetics/predisposition as an etiological category might reflect that they were taught this addiction model in treatment, but it is also possible that their CUDs were relatively and genuinely more influenced by genetic factors in light of their more frequent reports of family addiction problems [28]. There is indeed solid evidence that cannabis use and cannabis use disorders have heritable components [1, 33].

Participants were also asked to explain their understanding of why they were able to overcome their cannabis problem. The content analysis revealed that the top cited reasons were focusing on reasons for change, goal commitment to change, and conquering denial/self-deception. While there were no differences with respect to the recovery use status, treatment-assisted participants were more likely to perceive that they overcame their cannabis problem due to reasons of treatment/self-help and conquered underlying issues, whereas naturally recovered participants were more likely to cite the categories of focused on reasons for change, will power, and lost enjoyment/lifestyle change. These results suggest that participants attributed their recovery success to cognitive and motivational factors, which is consistent with our previous analyses demonstrating that cognitive strategies were the most helpful actions taken and maintenance factors involved in recovery [29]. These results support CBTMET approaches to the treatment of CUDs [7, 14], and might suggest that an increased cognitive and motivational focus might be one way to improve psychosocial treatments [29].

Finally, participants were asked to provide advice and recommendations to help another hypothetical person with a cannabis problem. The top cited advice categories were to seek help/social support, reflect on reasons for change, and engage in hobbies/distracting activities. Interestingly, no differences emerged between the abstinence- and moderation-oriented participants. Whereas treatment-assisted participants were more likely to endorse the category of seek help/social support naturally recovered participants were more likely to endorse the categories of reflect on reasons for change, engage in 
hobbies/distracting activities, and stimulus control/avoidance/change social environment. These results differ from the advice provided by a sample of recovered gamblers participating in a treatment outcome study [31], whereby it was mostly indicated that there was nothing that could be done to aid in the decision to cease or reduce gambling, with a smaller proportion suggesting awareness-raising strategies (e.g., pointing out the negative consequences of problem gambling and arousing cognitive dissonance).

The majority of participants reported that they would recommend treatment and self-help materials to a hypothetical person with a cannabis problem. However, treatment-assisted participants were more likely to recommend self-help materials compared to naturally recovered participants, whereas the latter group was more likely to recommend natural recovery (i.e., try to resolve the cannabis problem without professional assistance), albeit only among those who were abstinent. Interestingly, moderation status participants who were treatment-assisted were more likely to recommend natural recovery than other participants, which might reflect a perceived lack of fit between their goals and the goals of the treatment program they attended. It is unclear to what extent these recommendations might be influenced in the future if moderation-focused treatments for cannabis use disorders become widely available. Additionally, the majority of participants reported that they would recommend abstinence to a hypothetical person with a cannabis problem, and not surprisingly, moderation status participants were more likely to recommend moderation compared to abstinent status participants.

These findings are limited in that the results are based on self-reports of volunteer participants. Use of openended questions with probing by interviews, and qualitative analysis is helpful given how little is known about the recovery process. However, it is impossible to know how representative these individuals are of the general recovery population, and how able individuals are at identifying recovery influences. In addition, although the cannabis use histories were corroborated by family and friend interviews [18], the findings are based upon participant retrospective attributions of events occurring a number of years ago. Assessing similar issues earlier in recovery may elicit somewhat different information.

\section{Conclusions}

It is timely and important that we attend to how to best promote recovery from CUD. With the recent trend towards cannabis decriminalization and legalization, it remains unclear to what extent these legal changes might impact the incidence of cannabis use disorders. While the majority of individuals who use cannabis will not develop a problem, a substantial minority of individuals who will develop a problem deserve greater access to non-stigmatizing and improved treatment options. With a view towards informing evidence-based clinical practice, the present study explored the recovery process from cannabis use disorders in the context of multiple recovery pathways, the findings of which hold a number of implications for policy and practice. These insights from people with lived experience further support previous research that treatment-assisted and natural recoveries are for the most part similar with respect to the recovery process, which is consistent with Klingemann's contention that there exists a shrinking gap between the natural recovery and treatment outcome literature [23]. The frequent attribution of the use of cannabis for coping with negative emotions reinforces the inclusion of emotional regulation training in treatment protocols and prevention efforts. The results for cannabis are also consistent with those for other substances, which supports the growing attention to transdiagnostic etiological and treatment models focusing on common elements $[16,22$, 26].

However, participants, whether or not they had had treatment involvement, recommended the use of treatment and self-help materials to sharpen their focus on the reasons to change and to enhance their commitment to change. At the same time, they saw value in the efforts of individuals to recovery without help. As we have previously argued, these findings support the development of stepped care models that offer varying levels of support according to client wishes and needs [29]. Such models can include the provision of quality self-help materials by primary care providers and public health initiatives that promote self-directed efforts that are similar to treatment processes. The acknowledgement of moderation recovery outcomes within these models and self-help materials provides a more accurate and nuanced depiction of the recovery process, which might serve to de-stigmatize the non-isomorphic link between cannabis use and cannabis use disorder.

\section{Additional file}

Additional file 1. Content analyses category descriptions

Authors' contributions

This research was conducted as part of a dissertation conducted by JS, under the supervision of $\mathrm{DH}$. Both authors read and approved the final manuscript.

\section{Acknowledgements}

We appreciate the time and commitment of the volunteer participants.

Competing interests

The authors declare that they have no competing interests. 


\section{Availability of data and materials}

Available from the authors on reasonable request.

\section{Ethics approval}

Ethical approval was provided by the Conjoint Faculties Ethics Review Board at the University of Calgary.

\section{Publisher's Note}

Springer Nature remains neutral with regard to jurisdictional claims in published maps and institutional affiliations.

Received: 13 February 2018 Accepted: 14 July 2018

Published online: 30 July 2018

\section{References}

1. Agrawal A, Lynskey MT, Hinrichs A, Grucza R, Saccone SF, Krueger R, et al. A genome-wide association study of DSM-IV cannabis dependence. Addict Biol. 2011;16(3):514-8. https://doi.org/10.111 1/j.1369-1600.2010.00255.x.

2. American Psychiatric Association. Diagnostic and statistical manual of mental disorders. 5th ed. Washington, DC: Author; 2013.

3. Bischof G, Rumpf HJ, John U. Natural recovery from addiction. In: Shaffer HJ, LaPlante DA, Nelson SE, editors. APA addiction syndrome handbook, vol. 2. Washington, D.C.: American Psychologial Association; 2012. p. 133-55.

4. Boyd SJ, Tashkin DP, Huestis MA, Heishman SJ, Dermand JC, Simmons MS, Gorelick DA. Strategies for quitting among non-treatmentseeking marijuana smokers. Am J Addict. 2005;14(1):35-42. https://doi. org/10.1080/10550490590899835.

5. Braun V, Clarke V. Using thematic analysis in psychology. Qual Res Psychol. 2006;3:77-101.

6. Bujarski SJ, Norberg MM, Copeland J. The association between distress tolerance and cannabis use-related problems: the mediating and moderating roles of coping motives and gender. Addict Behav. 2012;37(10):1181-4. https://doi.org/10.1016/j.addbeh.2012.05.014.

7. Copeland J, Gates P, Pokorski I. A narrative review of psychological cannabis use treatments with and without pharmaceutical adjunct. Curr Pharm Des. 2016;22(42):6397-408. https://doi.org/10.2174/138161282266616 0831094811 .

8. Cunningham JA. Untreated remissions from drug use: the predominant pathway. Addict Behav. 1999;24(2):267-70.

9. Cunningham JA, Toneatto T, Koski-Jannes A. Why do people stop their drug use? Results from a general population survey. Contemp Drug Probl. 1999:26:695-710.

10. Dennis M, Babor TF, Roebuck MC, Donaldson J. Changing the focus: the case for recognizing and treating cannabis use disorders. Addiction. 2002;97:4-15.

11. Ellingstad TP, Sobell LC, Sobell MB, Eickleberry L, Golden CJ. Self-change: a pathway to cannabis abuse resolution. Addict Behav. 2006;31(3):519-30. https://doi.org/10.1016/j.addbeh.2005.05.033.

12. Foster KT, Arterberry BJ, lacono WG, McGue M, Hicks BM. Psychosocial functioning among regular cannabis users with and without cannabis use disorder. Psychol Med. 2017. https://doi.org/10.1017/S003329171 7003361.

13. Fox CL, Towe SL, Stephens RS, Walker DD, Roffman RA. Motives for cannabis use in high-risk adolescent users. Psychol Addict Behav. 2011;25(3):492-500. https://doi.org/10.1037/a0024331.

14. Gates PJ, Sabioni P, Copeland J, Le Foll B, Gowing L. Psychosocial interventions for cannabis use disorder. Cochrane Database Syst Rev. 2016:5:CD005336. https://doi.org/10.1002/14651858.cd005336.pub4

15. Gillespie NA, Neale MC, Legrand LN, lacono WG, McGue M. Are the symptoms of cannabis use disorder best accounted for by dimensional, categorical, or factor mixture models? A comparison of male and female young adults. Psychol Addict Behav. 2012;26(1):68-77. https://doi. org/10.1037/a0026230.
16. Griffiths MD. Components model of addiction within a biopsychosocial framework. J Subst Use. 2005;10:191-7. https://doi.org/10.1080/14659 890500114359.

17. Hasin DS, Grant B. NESARC findings on increased prevalence of marijuana use disorders-Consistent with other sources of information. JAMA Psychiatry. 2016;73(5):532. https://doi.org/10.1001/jamapsychiatry.2015.3158.

18. Hodgins DC, Stea JN. Psychometric evaluation of a lifetime version of the Marijuana Problems Scale. Addict Behav Rep. 2018;8:21-4. https://doi. org/10.1016/j.abrep.2018.05.001.

19. Johnston LD, Miech RA, O'Malley PM, Bachman JG, Schulenberg JE, Patrick ME. Monitoring the future. National survey results on drug use 1975-2017: overview. Key findings on adolescent drug use. Ann Arbor: Institute for Social Research, Univeristy of Michigan; 2018.

20. Kerridge BT, Mauro PM, Chou SP, Saha TD, Pickering RP, Fan AZ, et al. Predictors of treatment utilization and barriers to treatment utilization among individuals with lifetime cannabis use disorder in the United States. Drug Alcohol Depend. 2017;181:223-8. https://doi.org/10.1016/j. drugalcdep.2017.09.032

21. Kessler RC, Ustun TB. The world mental health (WMH) survey initiative version of the world health organization (WHO) composite international diagnostic interview (CIDI). Int J Methods Psychiatr Res. 2004;13(2):93-121.

22. Kim HS, Hodgins DC. Component model of addiction treatment: a pragmatic transdiagnostic treatment model of behavioral and substance addictions. Front Psychiatry. 2018 (in press).

23. Klingemann $\mathrm{H}$, Sobell MB, Sobell LC. Continuities and changes in selfchange research. Addiction. 2010;105(9):1510-8. https://doi.org/10.111 1/j.1360-0443.2009.02770.x

24. Nelson G, Macnaughton E, Curwood SE, Egalite N, Voronka J, Fleury MJ, et al. Collaboration and involvement of persons with lived experience in planning Canada's At Home/Chez Soi project. Health Soc Care Community. 2016;24(2):184-93. https://doi.org/10.1111/hsc.12197.

25. NVivo. NVivo qualitative data anlysis software. Version 9.0: QSR International Pty, Ltd. 2010

26. Shaffer HJ, LaPlante DA, Labrie JW, Kidman RC, Donato AN, Stanton MV. Toward a syndrome model of addiction: multiple expressions, common etiology. Harvard Rev Psychiatry. 2004;12:367-74

27. Simons J, Correia CJ, Carey KB, Borsari BE. Validating a five-factor marijuana motives measure: relations with use, problems, and alcohol motives. J Couns Psychol. 1998;45(3):265-73. https://doi. org/10.1037/0022-0167.45.3.265.

28. Stea JN (2013). Recovery from cannabis use disorders: abstinence versus moderation and treatment-assisted recovery versus natural recovery. (PhD), University of Calgary, Calgary.

29. Stea JN, Yakovenko I, Hodgins DC. Recovery from cannabis use disorders: abstinence versus moderation and treatment-assisted recovery versus natural recovery. Psychol Addict Behav. 2015;29(3):522-31. https://doi. org/10.1037/adb0000097.

30. Swift W, Hall W, Copeland J. One year follow-up of cannabis dependence among long-term users in Sydney, Australia. Drug Alcohol Depend. 2000:59(3):309-18.

31. Toneatto T, Dragonetti R. Effectiveness of community-based treatment for problem gambling: a quasi-experimental evaluation of cognitivebehavioral vs. twelve-step therapy. Am J Addict. 2008;17(4):298-303. https://doi.org/10.1080/10550490802138830.

32. United Nations Office on Drugs and Crime. 2014 world drug report. Vienna: United Nations; 2014

33. Verweij KJ, Zietsch BP, Liu JZ, Medland SE, Lynskey MT, Madden PA, et al. No association of candidate genes with cannabis use in a large sample of Australian twin families. Addict Biol. 2012;17(3):687-90. https://doi.org/10 1111/j.1369-1600.2011.00320.x. 